\title{
Improved thermoelectric performance in n-type half-Heusler NbCoSn by heavy-element Pt doping
}

\author{
Federico Serrano-Sánchez¹, Ting Luo², Junjie Yu³, Wenjie Xie ${ }^{4}$, Gudrun Auffermann ${ }^{1}$, Anke \\ Weidenkaff ${ }^{4}$, Tiejun Zhu ${ }^{3}$, Xinbing Zhao ${ }^{3}$, José A. Alonso ${ }^{5}$, Baptiste Gault ${ }^{2}$, Claudia Felser ${ }^{1}$, \\ Chenguang $\mathrm{Fu}^{1 *}$ \\ ${ }^{1}$ Max-Planck-Institut für Chemische Physik fester Stoffe, Nöthnitzer Str. 40, 01187 Dresden, Germany \\ 2Max-Planck-Institut für Eisenforschung GmbH, Max-Planck Straße 1, 40237, Düsseldorf, Germany \\ ${ }^{3}$ State Key Laboratory of Silicon Materials, School of Materials Science and Engineering, Zhejiang University, 310027 \\ Hangzhou, China \\ ${ }^{4}$ Department of Materials Science, Technical University of Darmstadt, 64287 Darmstadt, Germany \\ ${ }^{5}$ Instituto de Ciencias de Materiales de Madrid (ICMM). Consejo Superior de Investigaciones Científicas (CSIC). Sor \\ Juana Inés de la Cruz 3, 28049, Madrid, Spain \\ KEYWORDS: Half-Heusler, power factor, grain boundary, synchrotron XRD, SEM
}

\begin{abstract}
Half-Heusler compounds with a valence electron count of 18, including ZrNiSn, ZrCoSb, and NbFeSb, are good thermoelectric materials owing to favourable electronic structures. Previous computational studies had predicted a high electrical power factor in another half-Heusler compound $\mathrm{NbCoSn}$, but it has not been extensively investigated experimentally. Herein, the synthesis, structural characterization, and thermoelectric properties of the heavy-element Pt-doped NbCoSn compounds are reported. Pt is found to be an effective dopant enabling the optimization of electrical power factor, simultaneously leading to a strong point defect scattering of phonons, and thereby suppressing the lattice thermal conductivity. Annealing significantly improves the carrier mobility, which is ascribed to the decreased grain boundary scattering. As a result, a maximum power factor of $\sim 3.4 \mathrm{mWm}^{-1} \mathrm{~K}^{-2}$ is obtained at $600 \mathrm{~K}$. In conjunction with the reduced lattice thermal conductivity, a maximum figure of merit $z T$ of $\sim 0.6$ is achieved at $773 \mathrm{~K}$ for the post-annealed $\mathrm{NbCo}_{0.95} \mathrm{Pt}_{0.05} \mathrm{Sn}$, an increase of $100 \%$ compared to the undoped NbCoSn. This work highlights the important roles of the doping element and microstructure on the thermoelectric properties of half-Heusler compounds.
\end{abstract}

\section{Introduction}

Thermoelectric materials allow the direct conversion of heat into electrical energy. Thermoelectric devices are suitable for various applications owing to a lack of mobile parts, quiet operation, good reliability, endurance, and flexible implementation ${ }^{1,2}$. Therefore, they have been used for temperature control, power generation, and referigeration ${ }^{3-5}$. The conversion efficiency of a thermoelectric material is determined by its figure of merit, $z T$, which is calculated from the transport properties, i.e., $z T=\alpha^{2} \sigma T /\left(\kappa_{\mathrm{e}}+\kappa_{\mathrm{L}}\right)$, where $\alpha$ is the Seebeck coefficient, $\sigma$ is the electrical conductivity, $T$ is the absolute temperature, and $\kappa_{\mathrm{e}}$ and $\kappa_{\mathrm{L}}$ are the electronic and lattice components of thermal conductivity, respectively.

Among the strategies employed to improve the thermoelectric performance of a material, the optimization of carrier concentration $(n)$, usually through chemical doping or defect manipulation, could be the first and foremost one ${ }^{2}$. With this strategy, a large enhancement of $z T$ can be achieved if the initial $n$ of a material deviates significantly from its optimal one. Moreover, band engineering 6 and hierarchical phonon scattering ${ }^{7}$ are important approaches that can be implemented to further improve the thermoelectric performance 8,9 . For thermoelectric power generation devices, thermally stable, low-cost, and non-toxic materials are highly desirable ${ }^{8,10}$. Fulfilling these requirements, half-Heusler alloys with a valence electron count (VEC) of 18 exhibit good thermoelectric properties at high temperatures ${ }^{11,12}$ and have thus attracted considerable attention for power generation applications ${ }^{13,14}$. In the half-Heusler alloys, many different strategies have been employed to improve the thermoelectric performance besides carrier concentration optimization, e.g., point defect scattering ${ }^{15}$, nanostructuring ${ }^{16}$, phase separation ${ }^{17}$, band engineering ${ }^{18}$, and plastic deformation ${ }^{19}$. Consequently, several half-Heusler systems, including (Ti,Zr,Hf)NiSn ${ }^{17,20,21}, \quad(\mathrm{Ti}, \mathrm{Zr}, \mathrm{Hf}) \mathrm{CoSb}^{22-24}$, and $(\mathrm{V}, \mathrm{Nb}, \mathrm{Ta}) \mathrm{FeSb}^{19,25,26}$, have been found to exhibit good $z T$ values of above unity. Half-Heusler alloys with a nominal VEC of 19 have also attracted significant attention, where the intrinsic vacancies play a key role in determining the thermoelectric properties ${ }^{27}$.

$\mathrm{NbCoSn}$ is another half-Heusler alloy with a VEC of 18. The prediction of a large power factor, $\alpha^{2} \sigma$, in both n-type and p-type doped NbCoSn indicates promising thermoelectric properties ${ }^{28,29}$. Particularly, for the p-type NbCoSn, the calculated $\alpha^{2} \sigma$ is the largest among all studied half-Heusler alloys $^{28}$, which might originate from the high degeneracy in the valence band edge ${ }^{30}$. However, the preparation of a heavily hole-doped $\mathrm{NbCoSn}$ is experimentally challenging, 
and good thermoelectric performance has not been reported till date. Ti, Mo, and Hf were initially described as weak p-type acceptors for NbCoSn by Kawaharada et al. ${ }^{31,32}$ Later, Ferluccio et al. ${ }^{33}$ reported $\mathrm{Zr}$ - and Ti-doped NbCoSn with a positive Seebeck coefficient. Sc was also used as an acceptor for NbCoSn by Yan et al. ${ }^{34}$ However, the low electrical conductivities in these p-type NbCoSn samples suggested that the electrical power factors were not yet optimal, preventing the attainment of the predicted high performance. A possible hindrance in the realization of the heavily hole-doped $\mathrm{NbCoSn}$ could be the presence of interstitial Co, predicted to be the most stable intrinsic defect in $\mathrm{NbCoSn}$ by Bhattacharya and Madsen ${ }^{29}$, which could act as a 'killerdefect'. Excess Co was found in the synthesized $\mathrm{NbCoSn}$ samples, despite being designed to have the nominal composition $^{34,35}$. A similar phenomenon was also observed in the ZrNiSn system, in which excess Ni significantly changes the electronic structure and transport properties ${ }^{36,37}$. Therefore, further experimental understanding of the intrinsic defect in NbCoSn is necessary to optimize its p-type thermoelectric properties.

Meanwhile, n-type doping has been successfully achieved using $\mathrm{Sb}$ as a dopant ${ }^{31,33,38}$. The alloys of $\mathrm{NbCo}_{1+\mathrm{x}} \mathrm{Sn}$ were prepared by optical floating zone method ${ }^{39}$. Among these, $\mathrm{NbCo}_{1.05} \mathrm{Sn}$ showed the highest phase-purity and thermoelectric performance. He et al. ${ }^{40}$ reported $80 \%$ enhancement in the power factor by improving the samples' phase purity, resulting in a maximum $z T$ of 0.6 at $973 \mathrm{~K}$ in Sb-doped NbCoSn. Additionally, a material comprising the half-Heusler $\mathrm{NbCoSn}$ mixed with the full-Heusler $\mathrm{NbCo}_{2} \mathrm{Sn}$ was studied, and the antisite disorder was found to be suppressed by annealing ${ }^{41}$. Generally, half-Heusler alloys require a high content of chemical doping to realize the optimal electrical power factor and consequently, the dopants could also generate significant suppression on phonon transport ${ }^{13}$. A dopant element with large mass and radius differences compared to the host elements additionally increases phonon scattering, thereby suppressing the lattice thermal conductivity.

In this study, we have prepared heavy-element Pt-doped $\mathrm{NbCo}_{1-x} \mathrm{Pt}_{x} \mathrm{Sn}(x=0.00-0.15)$ samples using arc-melting and high-energy mechanical alloying, followed by a spark plasma sintering (SPS) process. After obtaining the sintered pellets, several samples are further annealed. Laboratory and synchrotron X-ray diffraction (XRD) measurements show that the obtained samples possess pure half-Heusler phases with negligible secondary phases. Heavy-element Pt doping leads to the simultaneously improved power factor and suppressed lattice thermal conductivity. Post-annealing is effective in decreasing the grain boundary scattering of carriers and thus, contributes to a high mobility. As a result, a maximum $z T$ of $\sim 0.6$ is obtained at $773 \mathrm{~K}$ for the postannealed $\mathrm{NbCo}_{0.95} \mathrm{Pt}_{0.05} \mathrm{Sn}$, which is the highest value for this system at this temperature.

\section{Experimental}

In the first set of the experiments, stoichiometric amounts of Nb slug (99.95\%, Alfa Aesar,), Co slug (99.95\%, Alfa Aesar), Sn shot (99.999\%, Alfa Aesar), and Pt shot (99.9\%) with compositions of $\mathrm{NbCo}_{1-x} \mathrm{Pt}_{x} \operatorname{Sn}(x=0.00,0.03,0.06,0.09$, 0.12 , and 0.15 ) were weighed and loaded in a water-cooled
$\mathrm{Cu}$ crucible of the arc-melter to prepare $4.5 \mathrm{~g}$ of each sample. Each pellet was melted three times under an inert $\mathrm{Ar}$ atmosphere to guarantee homogenization. To remove the residual impurities, the samples were annealed at $1073 \mathrm{~K}$ for seven days inside vacuum-sealed quartz ampoules using Ta foil to minimise the oxidation. Subsequently, the samples were ground using a ball-milling machine (Pulverisette 7, Fritsch), after which the resulting powders were compacted under inert conditions using SPS. The powders were placed in a $10-\mathrm{mm}$ cylindrical graphite die and a uniaxial pressure of $80 \mathrm{MPa}$ was applied while heating rapidly to $1223 \mathrm{~K}$ and then kept for $5 \mathrm{~min}$. Then, the sintered pellets were used for the characterization of structural and transport properties. In the second set of experiments, two additional $\mathrm{NbCo}_{1}$ ${ }_{x} \mathrm{Pt}_{x} \mathrm{Sn}$ samples were prepared with $x=0.05$ and 0.1 . To improve the phase purity, these two samples were additionally annealed for seven days at $1073 \mathrm{~K}$ after the SPS process.

XRD was performed at room temperature using an imageplate Huber G670 Guinier camera equipped with a Ge(111) monochromator and employing Co $\mathrm{K}_{\alpha 1}$ radiation. Synchrotron XRD (SXRD) patterns were collected with an incident beam of $28 \mathrm{keV}$ energy $(\lambda=0.4427 \AA)$, in the high angular resolution mode (MAD set-up) on the MSPD-diffractometer at the ALBA synchrotron in Barcelona, Spain. The ground samples were analysed using quartz capillaries with 0.25 $\mathrm{mm}$ radius. Rietveld refinements of XRD and SXRD patterns were performed using FullProf program ${ }^{42,43}$. A pseudoVoigt function was employed for analysing the shapes of the diffraction peaks. There were no excluded regions in the refinements. The final refinement of the synchrotron data was performed using parameters such as scale factor, set of background points, zero-point shift, pseudo-Voigt shape, and isotropic thermal displacement parameters of $\mathrm{Nb}$, $\mathrm{Co} / \mathrm{Pt}$, and Sn.

Microstructural analysis of the samples was performed using scanning electron microscopy (SEM, Zeiss Merlin scanning electron microscope, Carl Zeiss AG, Oberkochen, Germany) in the backscattered mode, at an accelerating voltage of $30 \mathrm{kV}$ and a beam current of $2 \mathrm{nA}$.

High-temperature measurements of the Seebeck coefficient and electrical resistivity were performed using an ULVAC-RICO ZEM-3 equipment. The thermal conductivity was calculated using the equation, $\kappa=C_{\mathrm{p}} D \rho$, where $C_{p}$ is the specific heat, $D$ is the thermal diffusivity, and $\rho$ is the sample density, employing thermal diffusivity values measured by a LASER Flash (LFA 457, Netzsch). $C_{\mathrm{p}}$ is calculated using the formula, $C_{\mathrm{p}}=C_{\mathrm{ph}, \mathrm{H}}+C_{\mathrm{D}}$, where $C_{\mathrm{ph}, \mathrm{H}}$ and $C_{\mathrm{D}}$ are the contributions of the harmonic phonons and lattice dilation, respectively, which can be calculated by employing the sound velocity, thermal expansion coefficient, and density (Table S1, supporting information) ${ }^{24}$.

The Hall effect measurements were performed in sample rods in a four-probe configuration using the resistivity option of the physical property measurement system (PPMS, Quantum Design). The measurements were symmetrised to eliminate the magnetoresistance contribution, and the carrier concentration was calculated using the formula, $R_{\mathrm{H}}=-$ $1 / e n_{\mathrm{H}}$, where $R_{\mathrm{H}}$ is the Hall coefficient, $e$ is the free electron charge, and $n_{\mathrm{H}}$ is the Hall carrier concentration.

\section{Results and Discussion}


The laboratory XRD patterns of the two sets of NbCo1${ }_{x} \mathrm{Pt}_{x} \operatorname{Sn}(x=0.00-0.15)$ samples are shown in Figure $1 \mathrm{a}$. The main phase is consistent with the cubic MgAgAs-type halfHeusler structure, and tiny impurity peaks are hardly observed in the range of binary phases $\left(\mathrm{Nb}_{3} \mathrm{Sn}, \mathrm{Co}_{7} \mathrm{Nb}_{6}\right)$ typically reported for this compound ${ }^{41}$. Although the impurity peaks are more noticeable for the samples with high Pt contents, the peak intensities are very low to enable precise identification of these secondary phases using the laboratory XRD analysis. Moreover, despite further annealing of the samples with $x=0.05$ and $x=0.10$ in the second set of experiments for seven days at $1073 \mathrm{~K}$, no marked difference in the phase purity is observed compared to those of the other samples from the first set of experiments. To perform a more detailed analysis of the structure and phase purity, high-resolution SXRD experiments were carried out for samples with $x=0.00$ and $x=0.05$ from the first and second sets of experiments, respectively. The crystalline structure corresponds to the $F \overline{4} 3 \mathrm{~m}$ space group, with $\mathrm{Nb}$ atoms located at $4 \mathrm{a}(0,0,0), \mathrm{Co} / \mathrm{Pt}$ at $4 \mathrm{c}(1 / 4,1 / 4,1 / 4)$, and $\mathrm{Sn}$ at $4 \mathrm{~b}$ $(1 / 2,1 / 2,1 / 2)$ Wyckoff positions. The refinements yield good agreement factors (Table S2, supporting information), while the isotropic displacement parameters are slightly higher than those described by neutron diffraction due to the effects of radiation absorption ${ }^{33,41}$. The accurate calculation of the occupation factors to analyze the defects as offstoichiometry or antisite defects is not possible owing to the highly symmetric positions of the atoms and the absorption effects. The full Heusler phase is not detected, and very weak intensity peaks are observed in the $9-11^{\circ}$ range corresponding to an extremely low volume fraction of the secondary impurity phases (Figures $1 b$ and $1 c$ ). Overall, the high-resolution SXRD measurements also suggest no marked differences in the phase purities for the samples with $x=0.00$ and $x=0.05$, despite the higher Pt content and post-annealing of the latter.

Lattice parameters (Figure 1d) were determined using Rietveld refinements of the XRD patterns in the $F \overline{4} 3 \mathrm{~m}$ space group, displaying an excellent match with the literature data $^{33}$. The lattice parameter value increases almost linearly with an increase in the Pt content, which indicates the efficiency of $\mathrm{Pt}$ as a donor for NbCoSn. Carrier concentration measurements allow the assessment of the effectiveness of Pt doping in terms of the electron transfer to the half-Heusler structure. Figure 1d shows a linear behaviour with an increase in the dopant concentration, and similar values have been reported for slightly higher Sb dopant concentrations ${ }^{40}$. Using a simple assumption of one added electron per Pt atom, the estimations of the carrier concentrations (red dashed line in Figure 1d) are consistent with the experimental values. However, carrier concentration is an integral measurement for a bulk sample, and the contribution of the possible micro- or nanoscale features within the material is compounded in the measured value. The calculated carrier concentration is $9.42 \times 10^{20} \mathrm{~cm}^{-3}$ for $x=0.05$, which is close to the experimental value. The results indicate that $\mathrm{Pt}$ is a highly effective dopant for this system.
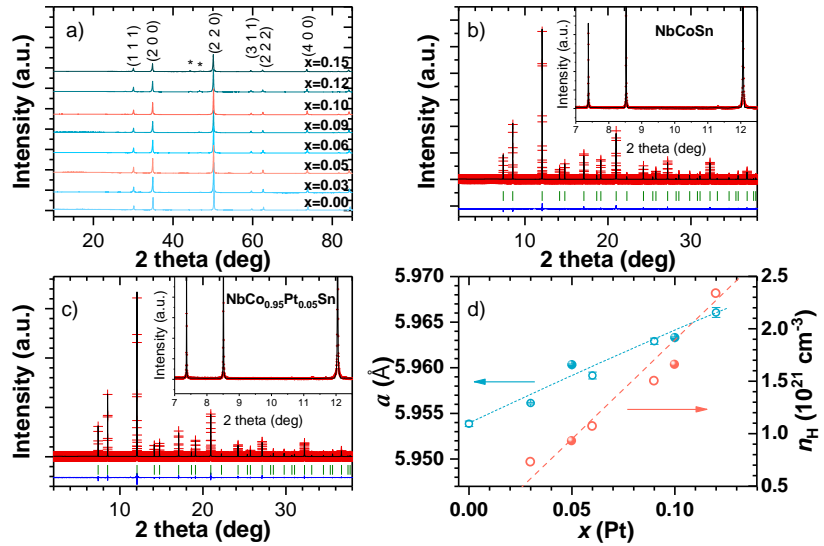

Figure 1. a) Indexed laboratory XRD patterns of of $\mathrm{NbCo}_{1-x} \mathrm{Pt}_{x} \mathrm{Sn}$ $(x=0.00-0.15)$. Rietveld refinement of synchrotron XRD patterns using $\lambda=0.4427 \AA$ at room temperature with experimental (red crosses), calculated (black line), difference (blue line), and Bragg reflections (green segments) of NbCoSn (b) and the post-annealed $\mathrm{NbCo0.95}_{0} \mathrm{Pt}_{0.05} \mathrm{Sn}$ (c), displaying an almost complete absence of impurities in the inset (magnified image) in the typical $2 \theta$ range. d) Lattice parameters for $\mathrm{NbCo}_{1-}$ ${ }_{x} \mathrm{Pt}_{x} \operatorname{Sn}(x=0.00,0.03,0.06,0.09,0.12$, and 0.15$)$ represented by empty blue circles, and post-annealed $\mathrm{NbCo}_{1-x} \mathrm{Pt}_{x} \mathrm{Sn}(x=0.05$, 0.10 ) represented by filled blue circles. Blue dashed line is a guide for the eye. Carrier concentration is represented by red empty and filled circles. The red dashed line denotes the carrier concentration calculated using one added electron per Pt atom.

The temperature-dependence of the electronic conductivity $\sigma$ for $\mathrm{NbCo}_{1-x} \mathrm{Pt}_{x} \mathrm{Sn}(x=0.00-0.15)$ samples is shown in Figure $2 \mathrm{a}$. An increasing trend is observed for the pristine $\mathrm{NbCoSn}$ sample, indicating a semiconducting behaviour. After doping with $\mathrm{Pt}$, the $\sigma$ value shows a noticeable increase in the complete temperature range, with a decreasing trend at temperatures above $500 \mathrm{~K}$, suggesting a degenerate semiconductor behaviour. Interestingly, the sample with $x=$ 0.05 , which is post-annealed, shows a higher $\sigma$ compared to that of the sample with $x=0.06$, despite the higher carrier concentration of the latter. A similar phenomenon is also observed for the other post-annealed sample with $x=0.10$, whose $\sigma$ value at room temperature is higher than that of the sample with $x=0.12$. Thereafter, the carrier mobilities of the samples were calculated (Figure 2b). Overall, the two post-annealed samples with $x=0.05$ and $x=0.10$ have higher carrier mobilities than those of the others, suggesting an annealing-induced change in the microstructure. Therefore, the backscattered SEM measurements were performed for samples with $x=0.05$ and $x=0.06$, as shown in Figures $2 \mathrm{c}$ and $2 \mathrm{~d}$, respectively. This analysis shows that the sample with $x=0.05$, with post-annealing after SPS, has a grain size of a few micrometres, which is almost ten times larger than that of the sample with $x=0.06$. Grain boundary scattering has recently been found to hinder the mobilities and conductivities (particularly near room temperature) of $\mathrm{Mg}_{3} \mathrm{Sb}_{2}{ }^{44}$, half-Heusler $\mathrm{Nb}_{1-x} \mathrm{Ti}_{x} \mathrm{FeSb}^{45}$ and (Zr,Hf)CoSb ${ }^{46}$, and elemental $\mathrm{Te}^{47,48}$. Herein, an increase in the grain size in post-annealed samples can support their high electrical conductivities and carrier mobilities. The confirmation of the influence of grain boundary processes will be the focus of a separate study. 

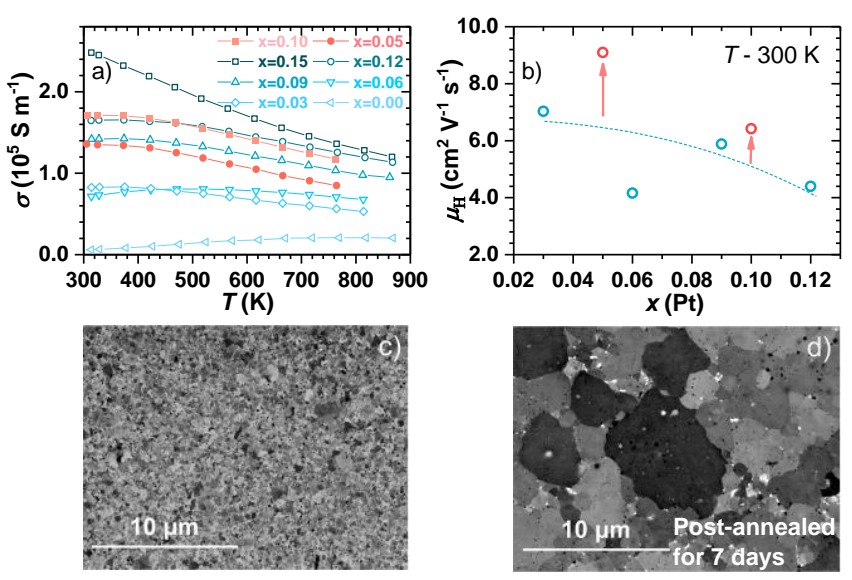

Figure 2. a) Temperature dependence of the electrical conductivity of $\mathrm{NbCo}_{1-x} \mathrm{Pt}_{x} \mathrm{Sn}(x=0.00-0.15)$. b) Carrier mobility as a function of the Pt content, where the dashed blue line is a guide for the eye. Backscattered SEM images of c) $\mathrm{NbCo0}_{0.94} \mathrm{Pt}_{0.06} \mathrm{Sn}$ and d) the post-annealed $\mathrm{NbCo}_{0.95} \mathrm{Pt}_{0.05} \mathrm{Sn}$.

The temperature dependence of the Seebeck coefficient (Figure 3a) of Pt-doped samples shows the typical behaviour of a degenerated semiconductor. The absolute Seebeck coefficient increases in the complete temperature range with no observable contribution from bipolar conduction. In contrast, the pristine $\mathrm{NbCoSn}$ shows an increase in the Seebeck coefficient up to $600 \mathrm{~K}$, when a small bump is observed. Similar data have been previously reported for the undoped compound ${ }^{33,39}$. As expected according to the Pisarenko relationship ${ }^{9}$, the absolute Seebeck coefficient decreases with an increase in the dopant content due to the increased electron concentration.

A Pisarenko plot using the single parabolic band model ${ }^{24}$ (Figure $3 \mathrm{~b}$ ) was used to better assess the electrical properties of n-type NbCoSn. A density of state effective mass $m^{*}$ of $6.5 m_{\mathrm{e}}$ is derived for the Pt-doped NbCoSn samples. Notably, this $m^{*}$ value is close to that of the n-type $(\mathrm{Zr}, \mathrm{Hf}) \mathrm{CoSb}^{24,46,49}$, but more than two times higher than that of $n$-type $(\mathrm{Zr}, \mathrm{Hf}) \mathrm{NiSn}^{20,50}$. As both $\mathrm{n}$-type ( $\left.\mathrm{Zr}, \mathrm{Hf}\right) \mathrm{CoSb}$ and $(\mathrm{Zr}, \mathrm{Hf}) \mathrm{NiSn}$ have a band degeneracy of 3 , and the calculated electronic structure of NbCoSn shows a higher band degeneracy of 6 ${ }^{28-}$ ${ }^{30}$, the band effective mass of NbCoSn is lower than that of ( $\mathrm{Zr}, \mathrm{Hf}) \mathrm{CoSb}$, but still higher than that of ( $\mathrm{Zr}, \mathrm{Hf}) \mathrm{NiSn}$.

The variation in calculated power factor is shown in Figure 3c. It follows an increasing trend with an increase in temperature because of an increase in the absolute Seebeck coefficient up to $600-700 \mathrm{~K}$ and then saturates at high temperatures due to the decreased electrical conductivity. $\mathrm{Pt}$ doped NbCoSn samples exhibit noticeably higher power factors than that of the undoped NbCoSn, suggesting the optimization of electrical properties. Moreover, the second set of samples with $x=0.05$ and $x=0.10$, which have larger grain sizes, show overall higher power factors than those of the first set of samples. This is attributed to the decreased grain scattering and thus improved carrier mobility. A maximum power factor of $\sim 3.4 \mathrm{mWm}^{-1} \mathrm{~K}^{-2}$ is obtained at $600 \mathrm{~K}$ for the post-annealed sample with $x=0.05$, which is comparable to that of $\mathrm{NbCoSn}_{0.9} \mathrm{Sb}_{0.1}$ at $900 \mathrm{~K}^{40}$.
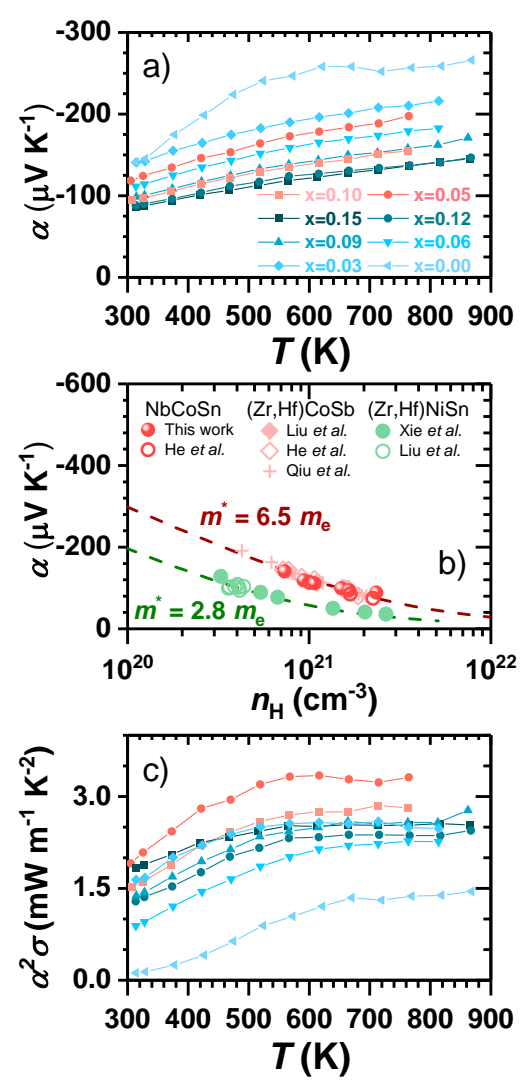

Figure 3.a) Temperature dependence of Seebeck coefficient for $\mathrm{NbCo}_{-x} \mathrm{Pt}_{x} \mathrm{Sn}(x=0.00-0.15)$. b) Pisarenko plot at room temperature for several n-type half-Heusler compounds including $\mathrm{NbCoSn}^{40}$, (Zr,Hf)CoSb $24,46,49$, and (Zr,Hf)NiSn ${ }^{20,50 . . ~ c) ~ T e m p e r a-~}$ ture dependence of power factor for $\mathrm{NbCo1-x}_{-x} \mathrm{Pt}_{x} \mathrm{Sn}(x=0.00$ $0.15)$.

The change in total thermal conductivity $\kappa$ of $\mathrm{NbCo}_{1-x} \mathrm{Pt}_{x} \mathrm{Sn}$ with temperature is shown in Figure 4a. A decrease in the $\kappa$ value of Pt-doped samples is observed near room temperature. To further understand this behaviour, $\kappa_{\mathrm{e}}$ and $\kappa_{\mathrm{L}}$ were calculated using the equations, $\kappa_{\mathrm{e}}=L \sigma T$ and $\kappa_{\mathrm{L}}=\kappa-\kappa_{\mathrm{e}}$, where $L$ is the Lorenz number and calculated using the single parabolic band model ${ }^{24}$. As shown in Figure $4 \mathrm{~b}$, a notable decrease in $\kappa_{\mathrm{L}}$ is observed upon Pt-doping and a maximum reduction of $45 \%$ is obtained for $x=0.15$, indicating that heavy element doping is effective in enhancing the phonon scattering by introducing strong mass and strain field fluctuations ${ }^{13}$. This reduction is almost linearly related to the $\mathrm{Pt}$ content as observed in Figure 4c, regardless of the grain sizes of the samples, suggesting that point defects dominate the phonon scattering while the grain boundaries might not significantly affect the phonon transport. At a high Pt content, $\kappa_{\mathrm{L}}$ shows lower values than those previously reported for this system, typically in the range of $5-10 \mathrm{~W} \mathrm{~m}^{-1} \mathrm{~K}^{-1}$ at room temperature ${ }^{33,40,41}$. In addition, an increase in $\kappa_{\mathrm{e}}$ is observed with an increase in the Pt content because of an increase in the electrical conductivity. Compared to Sb doping ${ }^{40}$, Pt doping is more effective at suppressing the $\kappa \mathrm{L}$ of $\mathrm{NbCoSn}$, thereby contributing to the decrease in $\kappa$. These values are comparable and even lower than those obtained 
for the $\mathrm{NbCoSn}-\mathrm{NbCoSb}$ solid-solutions, in which the disorder and $\mathrm{Nb}$ vacancies have a strong impact on the phonon conduction ${ }^{33}$.

Owing to the simultaneously decreased $\kappa_{\mathrm{L}}$ and improved power factor, Pt doping is effective in improving the figure of merit $z T$ of NbCoSn (Figure 4d). A $100 \%$ increase in the peak $z T$ is observed for $\mathrm{NbCo}_{0.95} \mathrm{Pt}_{0.05} \mathrm{Sn}$, compared to that for $\mathrm{NbCoSn}$, leading to a maximum value of $\sim 0.6$ at $773 \mathrm{~K}$. This value is similar to that for Sb-doped $\mathrm{NbCoSn}$ system but occurs at a lower temperature. Furthermore, the almost linear temperature dependence of $z T$ in all the studied $\mathrm{NbCo}_{1}$ ${ }_{x} \mathrm{Pt}_{x} \mathrm{Sn}$ samples suggests that a higher peak $z T$ can be achieved at higher temperatures.
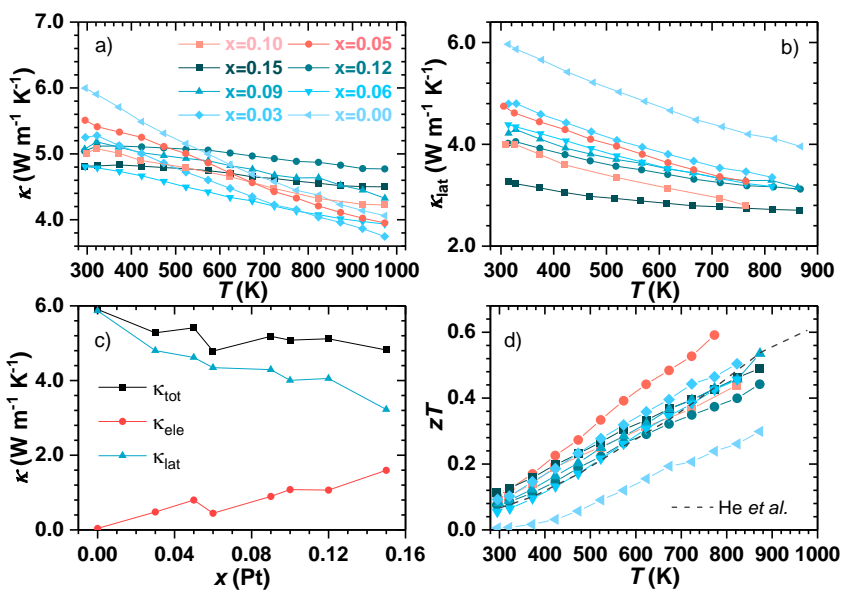

Figure 4. Temperature evolution of a) total thermal conductivity and b) lattice thermal conductivity. c) Variation of $\kappa, \kappa_{\mathrm{e}}$, and $\kappa_{\mathrm{L}}$ with an increase in Pt content at room temperature. d) Figure of merit $z T$ for $\mathrm{NbCo1-x}_{-x} \mathrm{Pt}_{x} \mathrm{Sn}(x=0.00-0.15)$ as a function of temperature. $z T$ for the optimised $\mathrm{NbCoSn}_{0.9} \mathrm{Sb}_{0.1}$ is also shown 40 .

\section{Conclusions}

In summary, heavy-element Pt-doped NbCoSn alloys are successfully prepared via arc-melting and high-energy mechanical alloying followed by SPS. The structure and thermoelectric properties are systematically characterized. Pt is found to be an effective dopant for $\mathrm{NbCoSn}$, leading to the simultaneously improved electrical and thermal transport properties, thereby resulting in enhanced thermoelectric performance. Post-annealing is effective in decreasing the grain boundary scattering of carriers and thus contributes to a high carrier mobility. A maximum power factor of $\sim 3.4$ $\mathrm{mWm}^{-1} \mathrm{~K}^{-2}$ is obtained at $600 \mathrm{~K}$. Furthermore, the lattice thermal conductivity is significantly decreased due to the enhanced point defect scattering of phonons, and a direct relationship between the doping content and this reduction is observed. A peak $z T$ of 0.6 at $773 \mathrm{~K}$ is obtained for the post-annealed $\mathrm{NbCo}_{0.95} \mathrm{Pt}_{0.05} \mathrm{Sn}$, which is comparable to the highest reported value and can be further improved at high temperatures. This work highlights the importance of the selection of doping elements and microstructure in improving the thermoelectric properties of half-Heusler compounds.

\section{ASSOCIATED CONTENT}

\section{Supporting Information.}

Calculated heat capacity, coefficient of linear thermal expansion, electronic contribution to thermal conductivity, structural parameters of Rietveld refinement of SXRD patterns. This material is available free of charge via the Internet at http://pubs.acs.org.

\section{AUTHOR INFORMATION}

\section{Corresponding Author}

*Chenguang.Fu@cpfs.mpg.de

\section{Author Contributions}

The manuscript was written through contributions of all authors. All authors have given approval to the final version of the manuscript.

Note

The authors declare no competing financial interest.

\section{ACKNOWLEDGMENT}

This work was funded by the Deutsche Forschungsgemeinschaft (DFG, German Research Foundation) - Projektnummer (392228380), the ERC Advanced Grant No. 742068 "TOPMAT", and the National Natural Science Foundation of China (no. 51761135127). F. Serrano acknowledges financial support by the Spanish Ministry of Economy and Competitivity through grant MAT2017-84496-R and the European Union's Horizon 2020 research and innovation program under the Marie Skłodowska-Curie grant agreement No 839821. Dr. T. Luo is grateful for the financial support from the Alexander von Humboldt Stiftung. We thank ALBA synchrotron (Barcelona, Spain) for providing the SXRD beam time. A. W. and W.X. acknowledge the financial support from Deutsche Forschungsgemeinschaft (Project No. BA 4171/4-1).

\section{REFERENCES}

(1) Rowe, D. M. Thermoelectrics, an Environmentally-Friendly Source of Electrical Power. Renew. Energy 1999, 16, 1251-1256.

(2) Snyder, G. J.; Toberer, E. S. Complex Thermoelectric Materials. Nat. Mater. 2008, 7, 105-114.

(3) Russ, B.; Glaudell, A.; Urban, J. J.; Chabinyc, M. L.; Segalman, R. A. Organic Thermoelectric Materials for Energy Harvesting and Temperature Control. Nat. Rev. Mater. 2016, 1, 16050.

He, R.; Schierning, G.; Nielsch, K. Thermoelectric Devices: A Review of Devices, Architectures, and Contact Optimization. Adv. Mater. Technol. 2018, 3, 1700256 .

Barnhart, T. R.; Ranalli, M.; Adldinger, M. Temperature Control Systems with Thermoelectric Devices. U.S. Patent No. 9,447,994, September 20, 2016.

(6) Pei, Y.; Shi, X.; LaLonde, A.; Wang, H.; Chen, L.; Snyder, G. J. Convergence of Electronic Bands for 
High Performance Bulk Thermoelectrics. Nature 2011, 473, 66-69.

Biswas, K.; He, J.; Blum, I. D.; Chun-IWu; Hogan, T. P.; Seidman, D. N.; Dravid, V. P.; Kanatzidis, M. G. High-Performance Bulk Thermoelectrics with All-Scale Hierarchical Architectures. Nature 2012, 489, 414-418.

(8) He, J.; Tritt, T. M. Advances in Thermoelectric Materials Research: Looking Back and Moving Forward. Science (80-. ). 2017, 357, eaak9997.

(9) Zhu, T.; Liu, Y.; Fu, C.; Heremans, J. P.; Snyder, J. G.; Zhao, X. Compromise and Synergy in HighEfficiency Thermoelectric Materials. Adv. Mater. 2017, 29.

(10) Rull-Bravo, M.; Moure, A.; Fernández, J. F.; Martín-González, M. Skutterudites as Thermoelectric Materials: Revisited. RSC Adv. 2015, 5, 41653-41667.

(11) Graf, T.; Felser, C.; Parkin, S. S. P. Simple Rules for the Understanding of Heusler Compounds. Prog. Solid State Chem. 2011, 39, 1-50.

Zhu, T.; Fu, C.; Xie, H.; Liu, Y.; Zhao, X. High Efficiency Half-Heusler Thermoelectric Materials for Energy Harvesting. Adv. Energy Mater. 2015, 5, 1500588.

(13) Fu, C.; Bai, S.; Liu, Y.; Tang, Y.; Chen, L.; Zhao, X.; Zhu, T. Realizing High Figure of Merit in HeavyBand p-Type Half-Heusler Thermoelectric Materials. Nat. Commun. 2015, 6, 8144.

(14) Xing, Y.; Liu, R.; Liao, J.; Zhang, Q.; Xia, X.; Wang, C.; Huang, H.; Chu, J.; Gu, M.; Zhu, T. HighEfficiency Half-Heusler Thermoelectric Modules Enabled by Self-Propagating Synthesis and Topologic Structure Optimization. Energy Environ. Sci. 2019, 12, 3390-3399.

(15) Yang, J.; Meisner, G. P.; Chen, L. Strain Field Fluctuation Effects on Lattice Thermal Conductivity of ZrNiSn-Based Thermoelectric Compounds. Appl. Phys. Lett. 2004, 85, 11401142.

(16) Xie, W.; Weidenkaff, A.; Tang, X.; Zhang, Q.; Poon, J.; Tritt, T. M. Recent Advances in Nanostructured Thermoelectric Half-Heusler Compounds. Nanomaterials 2012, 2, 379-412.

(17) Schwall, M.; Balke, B. Phase Separation as a Key to a Thermoelectric High Efficiency. Phys. Chem. Chem. Phys. 2013, 15, 1868-1872.

(18) Fu, C.; Zhu, T.; Liu, Y.; Xie, H.; Zhao, X. Band Engineering of High Performance P-Type FeNbSb Based Half-Heusler Thermoelectric Materials for Figure of Merit ZT > 1. Energy Environ. Sci. 2015, 8, 216-220.

(19) Rogl, G.; Ghosh, S.; Wang, L.; Bursik, J.; Grytsiv, A.; Kerber, M.; Bauer, E.; Mallik, R. C.; Chen, X.-Q.; Zehetbauer, M. Half-Heusler Alloys: Enhancement of ZT after Severe Plastic
Deformation (Ultra-Low Thermal Conductivity). Acta Mater. 2020, 183, 285-300.

(20) Liu, Y.; Xie, H.; Fu, C.; Snyder, G. J.; Zhao, X.; Zhu, T. Demonstration of a Phonon-Glass ElectronCrystal Strategy in (Hf, Zr) NiSn Half-Heusler Thermoelectric Materials by Alloying. J. Mater. Chem. A 2015, 3, 22716-22722.

(21) Rogl, G.; Sauerschnig, P.; Rykavets, Z.; Romaka, V. V; Heinrich, P.; Hinterleitner, B.; Grytsiv, A.; Bauer, E.; Rogl, P. (V, Nb)-Doped Half Heusler Alloys Based on $\{\mathrm{Ti}, \mathrm{Zr}, \mathrm{Hf}\}$ NiSn with High ZT. Acta Mater. 2017, 131, 336-348.

(22) Yan, X.; Liu, W.; Wang, H.; Chen, S.; Shiomi, J.; Esfarjani, K.; Wang, H.; Wang, D.; Chen, G.; Ren, Z. Stronger Phonon Scattering by Larger Differences in Atomic Mass and Size in P-Type Half-Heuslers Hf $1-\mathrm{x}$ Ti x CoSb 0.8 Sn 0.2. Energy Environ. Sci. 2012, 5, 7543-7548.

(23) Rausch, E.; Balke, B.; Ouardi, S.; Felser, C. Enhanced Thermoelectric Performance in the PType Half-Heusler (Ti/Zr/Hf) CoSb 0.8 Sn 0.2 System via Phase Separation. Phys. Chem. Chem. Phys. 2014, 16, 25258-25262.

(24) Liu, Y.; Fu, C.; Xia, K.; Yu, J.; Zhao, X.; Pan, H.; Felser, C.; Zhu, T. Lanthanide Contraction as a Design Factor for High-Performance HalfHeusler Thermoelectric Materials. Adv. Mater. 2018, 30, 1-7.

(25) Yu, J.; Fu, C.; Liu, Y.; Xia, K.; Aydemir, U.; Chasapis, T. C.; Snyder, G. J.; Zhao, X.; Zhu, T. Unique Role of Refractory Ta Alloying in Enhancing the Figure of Merit of NbFeSb Thermoelectric Materials. Adv. Energy Mater. 2018, 8, 1701313.

(26) Zhu, H.; Mao, J.; Li, Y.; Sun, J.; Wang, Y.; Zhu, Q.; Li, G.; Song, Q.; Zhou, J.; Fu, Y. Discovery of TaFeSbBased Half-Heuslers with High Thermoelectric Performance. Nat. Commun. 2019, 10, 1-8.

(27) Xia, K.; Liu, Y.; Anand, S.; Snyder, G. J.; Xin, J.; Yu, J.; Zhao, X.; Zhu, T. Enhanced Thermoelectric Performance in 18-Electron Nb0.8CoSb HalfHeusler Compound with Intrinsic Nb Vacancies. Adv. Funct. Mater. 2018, 28, 1-7.

(28) Yang, J.; Li, H.; Wu, T.; Zhang, W.; Chen, L.; Yang, J. Evaluation of Half-Heusler Compounds as Thermoelectric Materials Based on the Calculated Electrical Transport Properties. Adv. Funct. Mater. 2008, 18, 2880-2888.

(29) Bhattacharya, S.; Madsen, G. K. H. A Novel P-Type Half-Heusler from High-Throughput Transport and Defect Calculations. J. Mater. Chem. C 2016, 4, 11261-11268.

(30) Dylla, M. T.; Dunn, A.; Jain, A.; Snyder, G. J. Machine Learning Chemical Guidelines for Engineering Electronic Structures in HalfHeusler Thermoelectric Materials.

(31) KAWAHARADA, Y.; KUROSAKI, K.; MUTA, H.; 
UNO, M.; YAMANAKA, S. High Temperature Thermoelectric Properties of CoNb1-xHfxSn1-ySby Half-Heusler Compounds. J. Alloys Compd. 2004, 377, 312-315.

(32) Kawaharada, Y.; Kurosaki, K.; Muta, H.; Uno, M.; Yamanaka, S. High Temperature Thermoelectric Properties of CoNb1-XM XSn Half-Heusler Compounds. J. Alloys Compd. 2004, 384, 303-307.

(33) Ferluccio, D. A.; Smith, R. I.; Buckman, J.; Bos, J. W. G. Impact of $\mathrm{Nb}$ Vacancies and P-Type Doping of the NbCoSn-NbCoSb Half-Heusler Thermoelectrics. Phys. Chem. Chem. Phys. 2018, 20, 3979-3987.

(34) Yan, R.; Xie, W.; Balke, B.; Chen, G.; Weidenkaff, A. Realizing $\mathrm{p}$-Type $\mathrm{NbCoSn}$ Half-Heusler Compounds with Enhanced Thermoelectric Performance via Sc Substitution. Sci. Technol. Adv. Mater. 2020, 21, 122-130.

(35) Li, S.; Zhu, H.; Mao, J.; Feng, Z.; Li, X.; Chen, C.; Cao, F.; Liu, X.; Singh, D. J.; Ren, Z. N-Type TaCoSnBased Half-Heuslers as Promising Thermoelectric Materials. ACS Appl. Mater. Interfaces 2019, 11, 41321-41329.

(36) Zeier, W. G.; Schmitt, J.; Hautier, G.; Aydemir, U.; Gibbs, Z. M.; Felser, C.; Snyder, G. J. Engineering Half-Heusler Thermoelectric Materials Using Zintl Chemistry. Nat. Rev. Mater. 2016, 1, 16032.

(37) Fu, C.; Yao, M.; Chen, X.; Maulana, L. Z.; Li, X.; Yang, J.; Imasato, K.; Zhu, F.; Li, G.; Auffermann, G. Revealing the Intrinsic Electronic Structure of 3D Half-Heusler Thermoelectric Materials by AngleResolved Photoemission Spectroscopy. Adv. Sci. 2020, 7, 1902409.

(38) Ono, Y.; Inayama, S.; Adachi, H.; Kajitani, T. Thermoelectric Properties of NbCoSn-Based Half-Heusler Alloys. In 2006 25th International Conference on Thermoelectrics; 2006; pp 124127.

(39) Kimura, Y.; Tamura, Y.; Kita, T. Thermoelectric Properties of Directionally Solidified HalfHeusler Compound NbCoSn Alloys. Appl. Phys. Lett. 2008, 92, 2-5.

(40) He, R.; Huang, L.; Wang, Y.; Samsonidze, G.; Kozinsky, B.; Zhang, Q.; Ren, Z. Enhanced Thermoelectric Properties of N-Type NbCoSn Half-Heusler by Improving Phase Purity. APL Mater. 2016, 4.
Buffon, M. L. C.; Laurita, G.; Verma, N.;
Lamontagne, L.; Ghadbeigi, L.; Lloyd, D. L.; Sparks, T. D.; Pollock, T. M.; Seshadri, R. Enhancement of Thermoelectric Properties in the Nb-Co-Sn HalfHeusler/Heusler System through Spontaneous Inclusion of a Coherent Second Phase. J. Appl. Phys. 2016, 120.

(42) Rietveld, H. M. A Profile Refinement Method for Nuclear and Magnetic Structures. J. Appl. Crystallogr. 1969, 2, 65-71.

(43) Rodríguez-Carvajal, J. Recent Advances in Magnetic Structure Determination by Neutron Powder Diffraction. Phys. B 1993, 192, 55-69.

(44) Imasato, K.; Fu, C.; Pan, Y.; Wood, M.; Kuo, J. J.; Felser, C.; Snyder, G. J. Metallic N-Type Mg3Sb2 Single Crystals Demonstrate the Absence of Ionized Impurity Scattering and Enhanced Thermoelectric Performance. Adv. Mater. 2020, 1908218.

He, R.; Kraemer, D.; Mao, J.; Zeng, L.; Jie, Q.; Lan, Y.; Li, C.; Shuai, J.; Kim, H. S.; Liu, Y. Achieving High Power Factor and Output Power Density in PType Half-Heuslers Nb1-XTixFeSb. Proc. Natl. Acad. Sci. 2016, 113, 13576-13581.

Qiu, Q.; Liu, Y.; Xia, K.; Fang, T.; Yu, J.; Zhao, X.; Zhu, T. Grain Boundary Scattering of Charge Transport in N-Type (Hf, Zr) CoSb Half-Heusler Thermoelectric Materials. Adv. Energy Mater. 2019, 9, 1803447.

(47) Wu, Y.; Zhang, Q.; Liu, F.; Fang, T.; Zhu, T.; Zhao, X. Scattering Mechanisms and Compositional Optimization of High-Performance Elemental Te as a Thermoelectric Material. Adv. Electron. Mater. 2020, 2000038, 1-11.

Wu, Y.; Liu, F.; Zhang, Q.; Zhu, T.; Xia, K.; Zhao, X. B. Enhancing Average Thermoelectric Figure of Merit of Elemental Te by Suppressing the Grain Boundary Scattering. J. Mater. Chem. A 2020.

He, R.; Zhu, H.; Sun, J.; Mao, J.; Reith, H.; Chen, S.; Schierning, G.; Nielsch, K.; Ren, Z. Improved Thermoelectric Performance of N-Type HalfHeusler MCo1-XNixSb (M= Hf, Zr). Mater. Today Phys. 2017, 1, 24-30.

(50) Xie, H.; Wang, H.; Pei, Y.; Fu, C.; Liu, X.; Snyder, G. J.; Zhao, X.; Zhu, T. Beneficial Contribution of Alloy Disorder to Electron and Phonon Transport in Half-heusler Thermoelectric Materials. Adv. Funct. Mater. 2013, 23, 5123-5130. 
Table of Contents

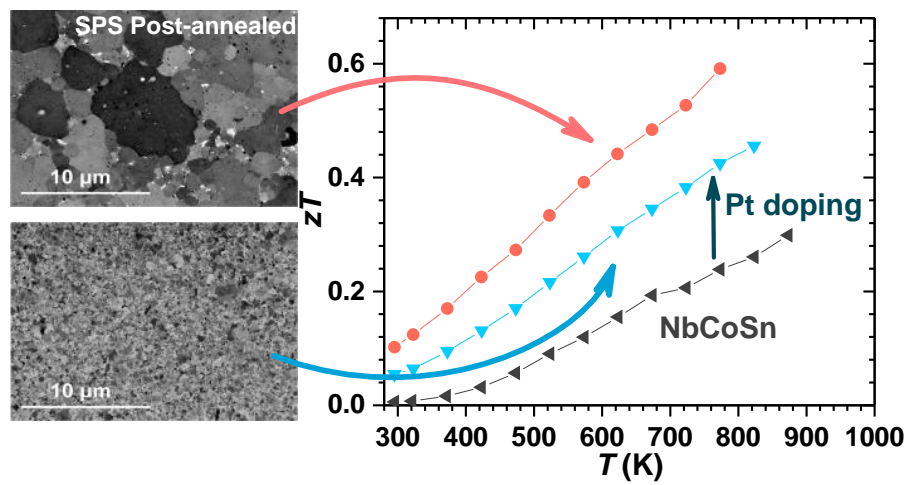




\section{Supporting Information}

\section{Improved thermoelectric performance in n-type half-Heusler NbCoSn by heavy-element Pt doping}

Federico Serrano-Sánchez ${ }^{1}$, Ting Luo ${ }^{2}$, Junjie $\mathrm{Yu}^{3}$, Wenjie Xie ${ }^{4}$, Gudrun Auffermann ${ }^{1}$, Anke Weidenkaff ${ }^{4}$, Tiejun Zhu ${ }^{3}$, Xinbing Zhao $^{3}$, José A. Alonso ${ }^{5}$, Baptiste Gault ${ }^{2}$, Claudia Felser ${ }^{1}$, Chenguang $\mathrm{Fu}^{1 *}$

${ }^{1}$ Max-Planck-Institut für Chemische Physik fester Stoffe, Nöthnitzer Str. 40, 01187 Dresden, Germany

${ }^{2}$ Max-Planck-Institut für Eisenforschung GmbH, Max-Planck Straße 1, 40237, Düsseldorf, Germany

${ }^{3}$ State Key Laboratory of Silicon Materials, School of Materials Science and Engineering, Zhejiang University, 310027

Hangzhou, China

${ }^{4}$ Department of Materials Science, Technical University of Darmstadt, 64287 Darmstadt, Germany

${ }^{5}$ Instituto de Ciencias de Materiales de Madrid (ICMM). Consejo Superior de Investigaciones Científicas (CSIC). Sor

Juana Inés de la Cruz 3, 28049, Madrid, Spain 
Table S1. Calculated and Dulong-Petit specific heat, longitudinal and transversal sound velocities, coefficient of linear thermal expansion (CLTE) and density for $x=0.05$ and $x=0.10 \mathrm{Pt}$-doped NbCoSn.

$\begin{array}{lllllll}\text { Composition } & \text { Calc. } C_{p} @ 300 K & D P C_{p} & v_{l} & v_{t} & \text { CLTE } & \rho \\ & \left(\mathrm{Jg}^{-1} \mathrm{~K}^{-1}\right) & \left(\mathrm{Jg}^{-1} \mathrm{~K}^{-1}\right) & \left(\mathrm{ms}^{-1}\right) & \left(\mathrm{ms}^{-1}\right) & \left(10^{-6} \mathrm{~K}^{-1}\right) & \left(\mathrm{gcm}^{-3}\right)\end{array}$

\begin{tabular}{l|llllll}
\hline NbCo $_{0.95} \mathbf{P t}_{\text {0.05 }}$ Sn & 0.252 & 0.269 & 5563 & 3028 & 9.14 & 8.64 \\
NbCo $_{0.9} \mathbf{P t}_{0.1}$ Sn & 0.246 & 0.263 & 5471 & 2906 & 10.7 & $(99.2 \%)$ \\
& & & & & & $(9.75$ \\
\end{tabular}

Figure S1. Calculated heat capacity from CLTE compared to Dulong-Petit value of Pt-doped NbCoSn.

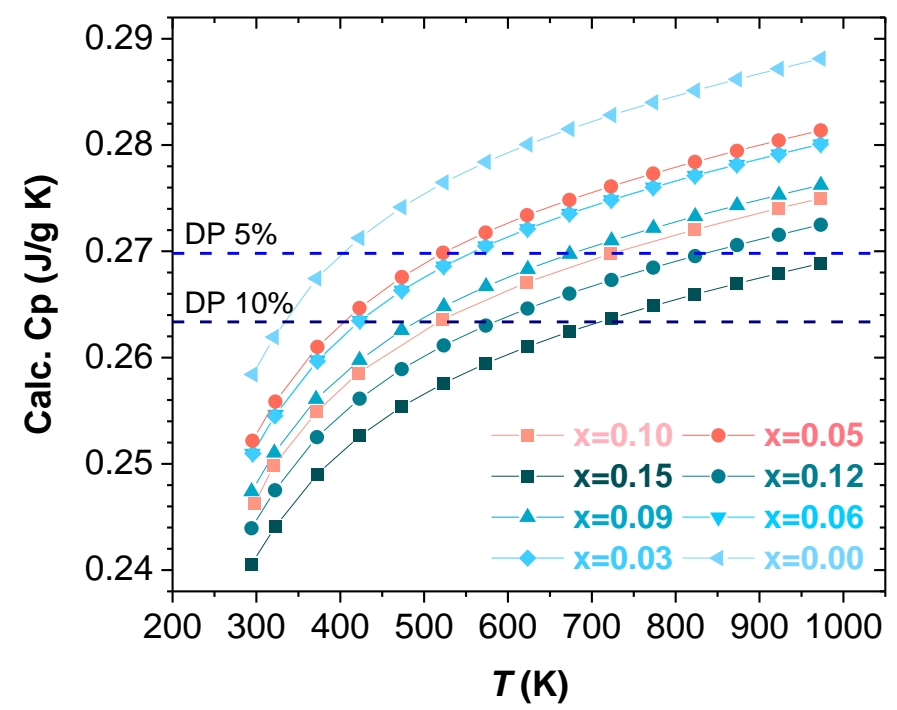


Figure S2. Electronic contribution to thermal conductivity of Pt-doped NbCoSn.

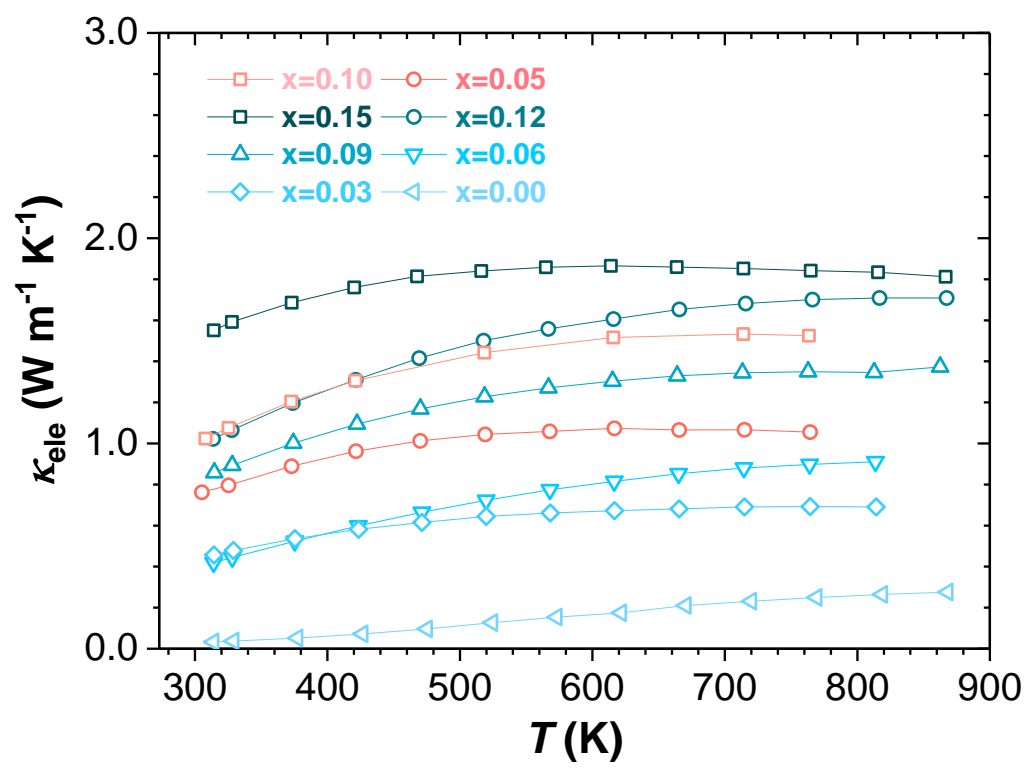

Table S2. Structural parameters of $\mathrm{NbCoSn}$ and $\mathrm{NbCo}_{0.95} \mathrm{Pt}_{0.05} \mathrm{Sn}$ obtained by Rietveld refinement of Synchrotron XRD patterns.

\begin{tabular}{|c|c|c|}
\hline Composition & $\mathrm{NbCoSn}$ & $N b C o_{0.95} P t_{0.05} S n$ \\
\hline Latt.parameter a/Å & $5.95386(4)$ & $5.96556(3)$ \\
\hline V/Å3 & $211.055(2)$ & $212.3020(17)$ \\
\hline $\mathrm{U}_{\text {iso }}, \mathbf{N b} / \AA^{2}$ & $0.0018(16)$ & $0.0041(13)$ \\
\hline $\mathbf{U}_{\text {iso, }}, \mathbf{C o} / \mathbf{P t} / \AA^{2}$ & $0.0054(7)$ & $0.0065(6)$ \\
\hline $\mathbf{U}_{\text {iso }}, \operatorname{Sn} / \AA^{2}$ & $0.0098(18)$ & $0.0106(14)$ \\
\hline $\mathbf{R}_{\mathbf{I}}$ & 3.15 & 2.06 \\
\hline $\mathbf{R}_{\mathbf{p}}$ & 6.18 & 9.42 \\
\hline $\mathbf{R}_{\mathrm{wp}}$ & 7.72 & 12.3 \\
\hline $\mathbf{R}_{\exp }$ & 4.97 & 7.27 \\
\hline$\chi^{2}$ & 2.41 & 2.87 \\
\hline
\end{tabular}

